rechnet werden. Dies gilt auch, wenn sich die Erbringung des präoperativen Komplexes gegebenenfalls in mehrere Sitzungen aufteilt.

Darüber hinaus ist bei Überweisung innerhalb derselben Arztgruppe zur ausschließlichen Durchführung des postoperativen Behandlungskomplexes nach Nr. 31600 EBM die Nr. 01436 EBM neben dem Komplex abrechenbar. Neben der Nr. 01436 ist für die Berechnung der Versichertenpauschale (EBM-Nrn. 03110-03130, 0411004130) in demselben Behandlungsfall mindestens ein weiterer persönlicher Arzt-Patienten-Kontakt notwendig. Dies bedeutet, dass die Nr. 01436 EBM nur dann anstelle der Versichertenpauschale im Überweisungsfall abzurechnen ist, wenn diese Zuweisung vor der kurativ hausärztlichen Behandlung des Quartals stattgefunden hat.

\title{
Was ist ein grober Behandlungsfehler?
}

- Der Bundesgerichtshof (BGH) hat entschieden, dass ein grober Behandlungsfehler vorliegt, wenn der Arzt gegen bewährte Behandlungsregeln oder gesicherte medizinische Erkenntnisse verstößt. Der Entscheidung (Urteil vom 25. Oktober 2011, Az.: VI ZR 139/10) lag der Fall einer Krankenhausärztin zu Grunde, die bei einem in der Nacht mit Myokardinfarkt eingelieferten Patienten keine sofortige Fibrinolyse anordnete. In der Folge kam es zu irreversiblen Schädigungen des Herzmuskelgewebes.

Der Patient klagte daraufhin gegen die Ärztin auf Schmerzensgeld und Schadenser- satz. Das Berufungsgericht lehnte die Klage ab, weil nach Ausführungen des Sachverständigen kein grober Behandlungsfehler festgestellt werden konnte. Der angerufene BGH wollte dieser Entscheidung aber nicht folgen. Die Richter urteilten, dass bei dieser Sachlage das Berufungsgericht die Wertung des Sachverständigen, das eindeutig fehlerhafte Vorgehen der Beklagten sei noch verständlich, nicht ohne weiteres übernehmen durfte. Der Sachverständige hatte darauf hingewiesen, dass Anhaltspunkte für eine Wiedereröffnung der verschlossenen Herzkranzgefäße nicht gegeben waren. Bei dieser Sachlage liege es nahe, dass der Sach-

\section{MMW Kommentar}

Konkret bedeutet das: Wird der Patient im Quartal bereits betreut und die Versichertenpauschale wurde bereits abgerechnet, kann die Nr. 01436 EBM nicht mehr angesetzt werden. Folgekontakte sind dann Teilleistungen der Versichertenpauschale. Im umgekehrten Fall ist dies allerdings möglich.

verständige bei der Bewertung des Gewichts des ärztlichen Fehlverhaltens maßgeblich auf den Grad der subjektiven Vorwerfbarkeit abgestellt hatte.

Auf die subjektive Vorwerfbarkeit komme es aber nicht an. Erforderlich, aber auch genügend sei vielmehr ein Fehlverhalten, das nicht aus subjektiven, in der Person des handelnden Arztes liegenden Gründen, sondern aus objektiver ärztlicher Sicht nicht mehr verständlich erscheint. Hierauf hätte das Berufungsgericht nach Auffassung der Bundesrichter den Sachverständigen hinweisen und seine Einschätzung kritisch hinterfragen müssen.

\section{MMW Kommentar}

Der Trend der vergangenen Jahre zu einer Verschärfung der Arzthaftungsregeln setzt sich mit dieser Entscheidung weiter fort. Es wird deshalb immer wichtiger, dass die Dokumentation der Aufklärung selbst, aber auch die Durchführung einzelner Maßnahmen nicht nur fragmentarisch, sondern vielmehr umfassend erfolgt. Nur so ist zu gewährleisten, dass man im Haftungsfall ausreichend geschützt ist. Dies muss umso mehr Beachtung für die Fälle finden, in denen es klare Behandlungsrichtlinien gibt. Nach Auffassung des BGH ist nämlich ein Behandlungsfehler als grob zu bewerten, wenn der Arzt eindeutig gegen bewährte ärztliche Behandlungsregeln oder gesicherte medizinische Erkenntnisse verstoßen und einen Fehler begangen hat, der aus objektiver Sicht nicht mehr verständlich erscheint, weil er einem Arzt schlechterdings nicht unterlaufen darf.

In diesem Zusammenhang ist auch das am 1.1.2013 in Kraft tretende neue Patientenrechtegesetz zu sehen. Ziel dieser Gesetzesinitiative ist es, das Informationsgefälle zwischen Arzt und Patient auszugleichen. Patienten müssen künftig über die Behandlung umfassend informiert werden. Bei Streitigkeiten soll die Patientenakte das wichtigste Dokument werden, denn bei groben
Behandlungsfehlern muss der Arzt beweisen, dass die Behandlung auch ohne den Fehler schief gelaufen wäre.

Näher betrachtet muss man zwar eher zu der Erkenntnis kommen, dass dieses neue Gesetz mehr den spezialisierten fachärztlichen Bereich tangiert. Ein Punkt aus der Gesetzesneuerung dürfte allerdings auch für den Hausarzt wichtig sein: Was ist der Unterschied zwischen einem "einfachen" und einem "groben" Behandlungsfehler? Diesbezüglich gibt es bisher nämlich keine einheitliche Rechtsprechung. 